\title{
Carotid Webs: Radiographic Appearance and Significance
}

\author{
Kyle Wojcik, MS, ${ }^{1}$ James Milburn, MD, ${ }^{1,2}$ Gabriel Vidal, MD, ${ }^{1,3}$ Andrew Steven, MD ${ }^{1,2}$ \\ ${ }^{1}$ The University of Queensland School of Medicine, Ochsner Clinical School, New Orleans, LA ${ }^{2}$ Department of Radiology, Ochsner Clinic \\ Foundation, New Orleans, LA ${ }^{3}$ Department of Neurology, Ochsner Clinic Foundation, New Orleans, LA
}

\section{INTRODUCTION}

First identified and named in 1973, carotid webs are nonatherosclerotic fibrous bands that arise along the posterior margin of the carotid bulb. ${ }^{1,2}$ Histopathologically, these entities are characterized by fibroelastic thickening of the arterial intima. They appear on imaging modalities as shelf-like or triangular-shaped intraluminal projections. The prevalence of these lesions is estimated to be on the order of $1 \%$, although they may be overlooked in clinical practice as they are not typically associated with significant vascular stenosis.

The significance of these lesions is not yet known, but evidence is emerging for an association with ischemic strokes. ${ }^{3-7}$ Up to one-third of all patients presenting with ischemic strokes lack an identifiable cause, and the strokes are classified as cryptogenic in etiology, with most cases occurring in younger patients without traditional vascular risk factors. ${ }^{8}$ Carotid webs are suspected to be an underdiagnosed cause of cryptogenic strokes and are thought to serve as a nidus for thrombus formation.
We present an image-rich review of 5 recent stroke patients who presented to Ochsner Health System and were found to have an ipsilateral carotid web. We review the imaging features and patient management for each case.

\section{CASE 1}

History

A 45-year-old female with a medical history of right middle cerebral artery (MCA) syndrome in 2015 (treated with tissue plasminogen activator [tPA] with complete resolution) presented as a transfer from an outside hospital to Ochsner with a 10-hour history of left-sided weakness, left facial droop, and lethargy (no tPA was administered during this admission). Computed tomography angiography (CTA) on admission (Figure 1A, 1B, 1C) demonstrated subtle irregularity along the posterior margin of right internal carotid artery origin, a typical appearance for a carotid web. Diffusionweighted imaging (DWI) and apparent diffusion coefficient on admission (Figure 2B) demostrated a new right MCA infarct. Previous fluid attenuation inversion recovery (FLAIR)
A

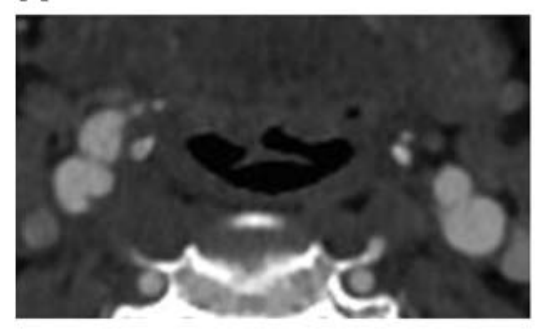

B

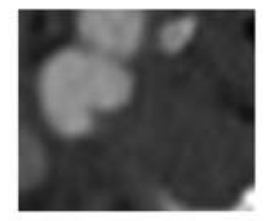

C

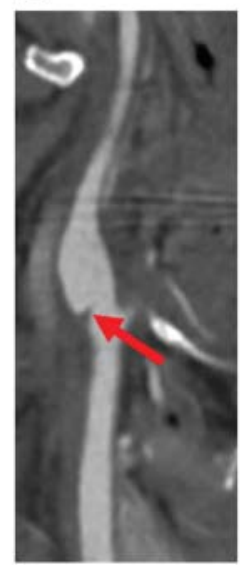

D

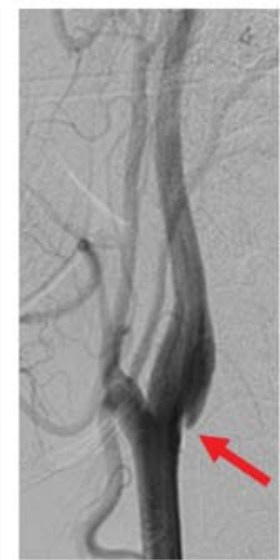

Figure 1. Case 1, a 45-year-old female with recurrent small strokes in the right middle cerebral artery. (A) Axial computed tomography angiography (CTA) images on admission demonstrate a subtle irregularity along the posterior margin of right internal carotid artery origin, a typical appearance for a carotid web. (B) Magnified image from Figure 1A further illustrates the triangular intraluminal band along the posterior margin of the right internal carotid artery. (C) Coronal reconstruction of the CTA and (D) digital subtraction angiogram exhibit the characteristic shelf-like filling defect at the level of the carotid bulb (arrows). Note the lack of significant stenosis $(<50 \%)$. 
A

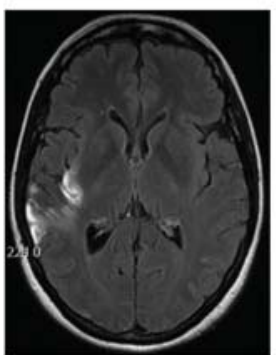

B

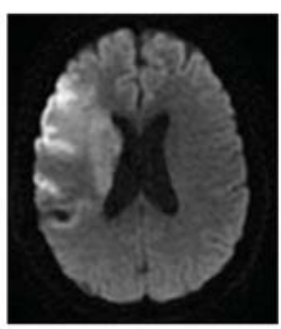

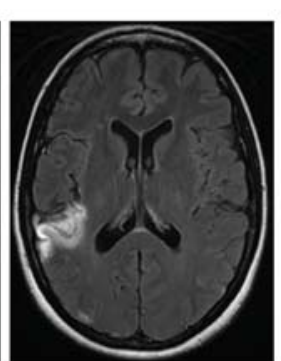

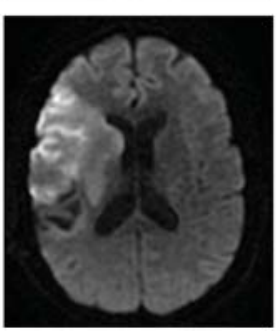

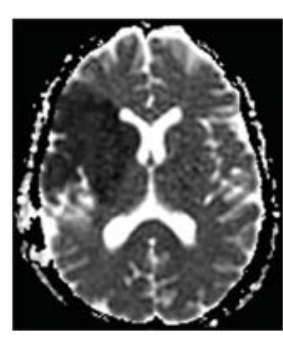

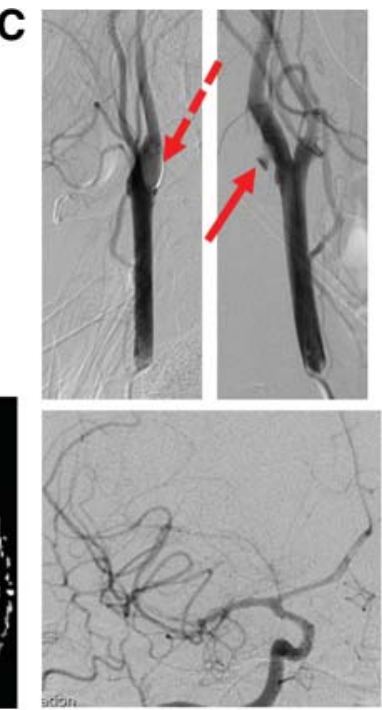

Figure 2. Case 1, a 45-year-old female (same patient shown in Figure 1 images) with recurrent infarctions in the right middle cerebral artery. (A) Axial fluid attenuation inversion recovery (FLAIR) images demonstrate areas of prior right middle cerebral artery infarction, including the posterior insular cortex and temporal lobe. (B) Diffusion-weighted imaging and apparent diffusion coefficient on admission show a new acute infarct, also in right middle cerebral artery territory, now involving the basal ganglia and opercular frontal lobe. (C) Digital subtraction angiogram images show an intraluminal thrombus along the posterior margin of the right carotid bulb (solid arrow) and an embolus in the anterior M2 division of the right middle cerebral artery (dashed arrow).

imaging demonstrated areas of prior right MCA infarcts (Figure 2A).

\section{Management}

The patient did not fit the criteria for tPA or mechanical thrombectomy and was started on aspirin $325 \mathrm{mg}$ daily, heparin 5,000 units subcutaneously every 8 hours, and atorvastatin $40 \mathrm{mg}$ daily. Five days later, diagnostic cerebral angiogram (Figure 1D, 2C) demonstrated a shelf-like filling defect at the level of the carotid bulb. Two days later, the patient was discharged to a rehabilitation facility on aspirin $325 \mathrm{mg}$ daily, clopidogrel $75 \mathrm{mg}$ daily, enoxaparin sodium (Lovenox) $0.40 \mathrm{~mL}(40 \mathrm{mg})$ subcutaneously twice daily, and atorvastatin $40 \mathrm{mg}$ daily. Two months later, after the patient's left-sided weakness had improved, right carotid endarterectomy and bovine patch angioplasty were performed. Pathology of the endarterectomy was reported to be an intimal form of fibromuscular dysplasia in the carotid web. The patient was discharged on aspirin $325 \mathrm{mg}$ daily, clopidogrel $75 \mathrm{mg}$ daily, and atorvastatin $40 \mathrm{mg}$ daily, with reduction to single antiplatelet aspirin $325 \mathrm{mg}$ daily after 2 months. Approximately 1 year later, the patient had residual mild speech difficulty and left-sided weakness.

\section{CASE 2}

\section{History}

A 44-year-old female with no stroke history presented to an outside hospital with mild headache, nausea, and acute onset of left hemiplegia with facial droop. The patient was administered tPA and transferred to Ochsner for possible endovascular intervention. On admission, CTA (Figure
$3 \mathrm{~A}, 3 \mathrm{~B})$ demonstrated an abrupt filling defect of the right carotid bifurcation.

\section{Management}

The patient underwent emergent endovascular treatment with mechanical thrombectomy of a distal internal carotid artery thrombus (Figure 3C, 3D) and placement of a vascular stent across the web (Figure 3E). Follow-up head CT (Figure 3F) demonstrated a small infarct in the right lentiform nucleus. The patient showed marked improvement from her initial presentation of left hemiplegia and neglect, with no residual deficit or recurrent symptoms. Follow-up care included dual antiplatelet therapy of aspirin $325 \mathrm{mg}$ and clopidogrel $75 \mathrm{mg}$ daily with atorvastatin $40 \mathrm{mg}$ daily for 3 months, followed by only aspirin $325 \mathrm{mg}$ and atorvastatin $40 \mathrm{mg}$ daily. At 4-month follow-up, conventional angiogram showed patent right proximal internal carotid artery stent without in-stent stenosis.

\section{CASE 3}

History

A 52-year-old female presented to an outside emergency department (ED) with acute onset of slurred speech, right facial droop, confusion, and word finding difficulty. In the ED, tPA was administered with reported complete resolution of symptoms within 30 minutes. The patient was transferred to Ochsner for further evaluation. Head magnetic resonance imaging (MRI) (Figure $4 \mathrm{~A}$ ) demonstrated acute infarction, and CTA (Figure 4B) demonstrated a shelf-like defect of the left carotid bifurcation consistent with a carotid web. 
A

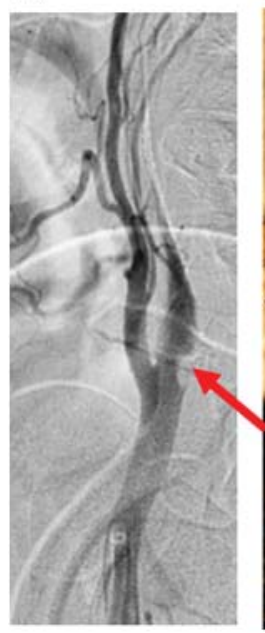

B

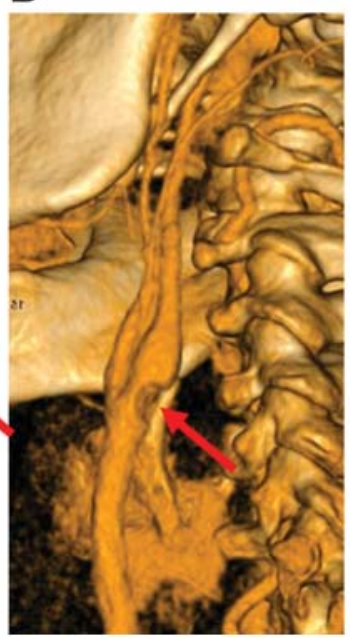

C

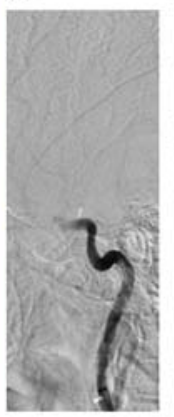

D

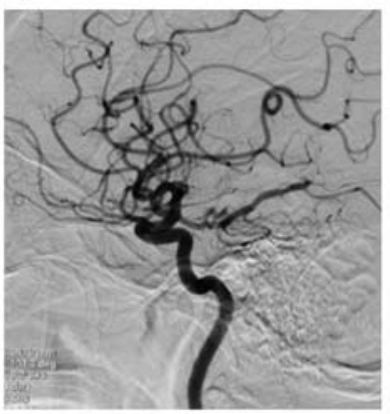

E

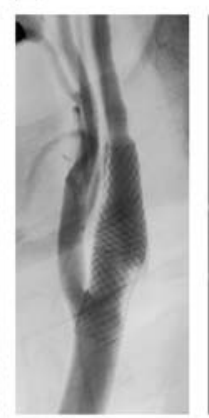

F

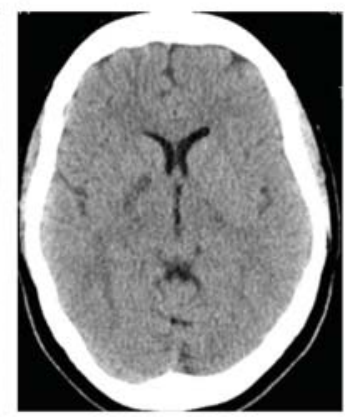

Figure 3. Case 2, a 44-year-old female with acute right middle cerebral artery stroke. (A) Digital subtraction angiogram and (B) surface 3-dimensional reconstruction of computed tomography angiography (CTA) show an abrupt filling defect (arrow) along the posterior margin of the right carotid bifurcation. Digital subtraction angiograms (C) before and (D) after mechanical thrombectomy of the distal internal carotid artery occlusion show return of blood flow. (E) Posttreatment digital subtraction angiogram shows the WALLSTENT (Boston Scientific Corp.) deployed over the carotid web. (F) Follow-up noncontrast head CT shows only a small infarct in the right lentiform nucleus.

A
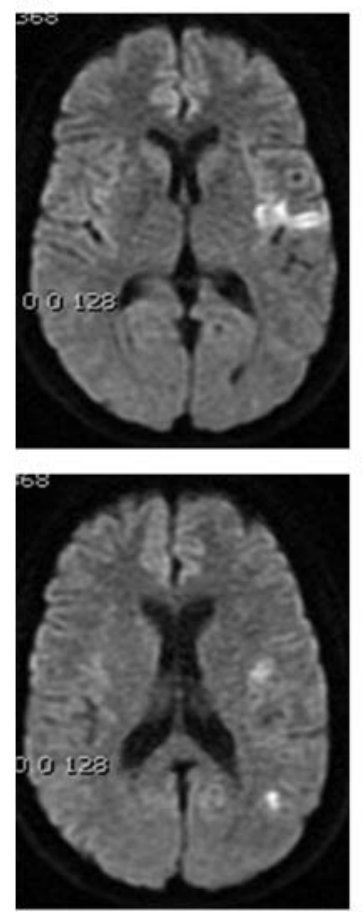

B
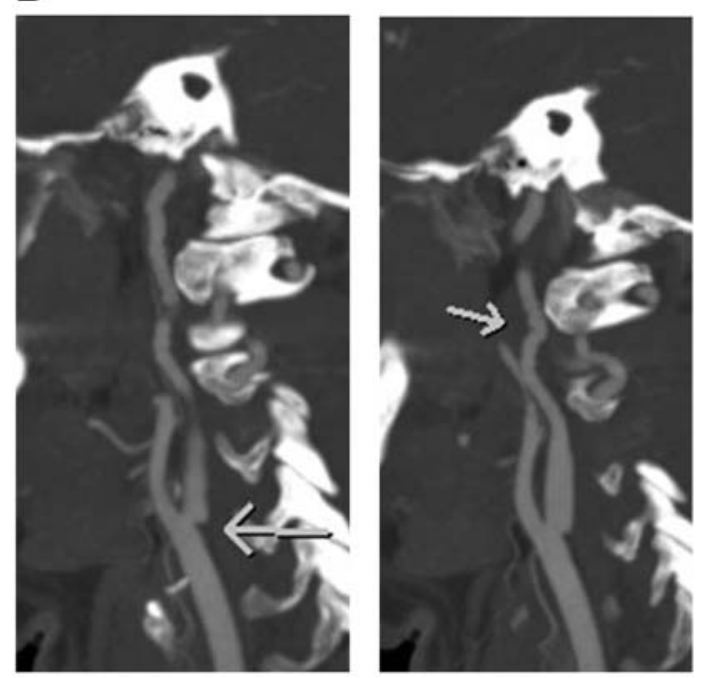

Figure 4. Case 3, a 52-year-old female with acute middle cerebral artery stroke. (A) Axial diffusion-weighted images show small regions of diffusion restriction consistent with acute infarction within the left middle cerebral artery distribution, including the insula and posterior temporal lobe. (B) Sagittal reconstructions from concurrent computed tomography angiography (CTA) show a nonstenotic shelf-like defect (long arrow) along the posterior margin of the left carotid bifurcation consistent with a carotid web. Beaded irregularity elsewhere in the cervical segment of the internal carotid artery indicates underlying fibromuscular dysplasia (short arrow). 
A

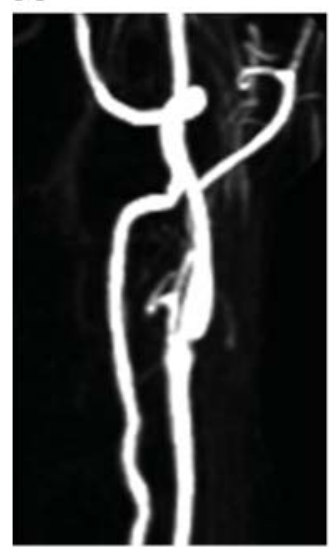

B

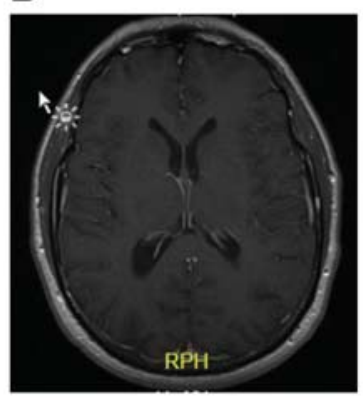

C

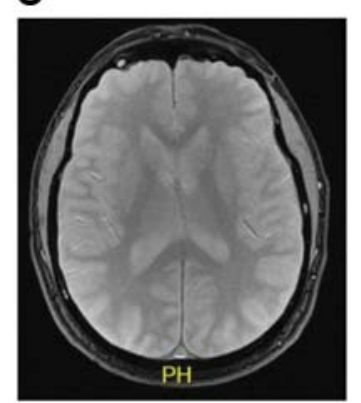

Figure 5. Case 4, a 47-year-old male with transient ischemic attack. (A) Maximum intensity projection reformat of a contrast-enhanced magnetic resonance angiogram of the neck shows a shelf-like projection along the posterior wall of the proximal left internal carotid artery just distal to the bifurcation and with minimal stenosis. (B) Axial T1 and (C) T2 images of the brain show no evidence of recent infarction.

\section{Management}

The patient was monitored in the intensive care unit for 24 hours post-tPA and was discharged with medical management (aspirin $81 \mathrm{mg}$, clopidogrel $75 \mathrm{mg}$, and atorvastatin $40 \mathrm{mg}$ daily). No surgical or endovascular intervention was performed.

\section{CASE 4}

\section{History}

A 47-year-old male with a history of hyperlipidemia presented with acute onset left facial numbness/tingling and left hand tingling. Magnetic resonance angiogram (MRA) (Figure 5A) demonstrated a shelf-like projection of the left internal carotid artery. MRI brain did not show any evidence of recent stroke (Figure 5B, 5C).

\section{Management}

The patient's symptoms resolved spontaneously with no interventions, and transient ischemic attack (TIA) was diagnosed. The patient was discharged with medical management (aspirin $81 \mathrm{mg}$, clopidogrel $75 \mathrm{mg}$, and atorvastatin $40 \mathrm{mg}$ daily, but the atorvastatin was later changed to simvastatin $20 \mathrm{mg}$ because of myalgia complications with atorvastatin). At 2-month follow-up, the patient reported no ongoing or new symptoms.

\section{CASE 5}

\section{History}

A 51-year-old female with a medical history of hyperlipidemia and lymphoma (in remission) presented with acute onset right-sided weakness, aphasia, hemianopsia, and facial droop. The patient received tPA at an outside facility with reported mild improvement in physical examination and was transferred to Ochsner for possible endovascular intervention. CTA (not shown) confirmed occlusion of the left MCA. MRI revealed an acute infarct (Figure 6B, 6C).

\section{Management}

The patient was treated successfully with aspiration thrombectomy, during which a shelf-like filling defect of the left internal carotid artery was noted (Figure 6A). The patient was discharged on dual antiplatelet therapy with mild residual right upper extremity weakness, facial droop, hemianopsia, and aphasia. Approximately 1 week later, the patient returned as an outpatient for stenting of the left internal carotid artery web and was continued on clopidogrel $75 \mathrm{mg}$ daily for 1 month and aspirin $325 \mathrm{mg}$ daily indefinitely.

At 4-month follow-up, the previously placed left carotid stent had $10 \%$ diameter stenosis within the proximal portion. The right carotid injections showed that the patient also had a small, thin contralateral carotid web without significant stenosis that was not deemed appropriate for stenting. Continued management included aspirin $325 \mathrm{mg}$, clopidogrel $75 \mathrm{mg}$, and atorvastatin $10 \mathrm{mg}$ daily.

\section{UNIQUE IMAGING FEATURES OF CAROTID WEBS}

Carotid webs exhibit a unique shape and location. They appear as triangular, thin filling defects most commonly arising from the posterior aspect of the proximal internal carotid artery. Given their small size (generally $1-2 \mathrm{~mm}$ in thickness) and orientation, they can be quite subtle, especially on axial imaging. Thus, multiplanar reformats (oblique sagittal images) are best for identification with CTA or MRA. Multiplanar reconstructions allow for 3-dimensional analysis of the characteristic filling defect and clearly distinguish this pathology from similar pathologies such as fibromuscular dysplasia and atherosclerosis. Fibromuscular dysplasia-a segmental, noninflammatory, nonatherosclerotic vascular disease of small- to medium-sized arteries-most commonly affects middle and distal portions of the internal carotid and vertebral arteries and appears as multiple stenoses in a string-of-beads pattern that is distinct from carotid webs. ${ }^{9,10}$ Additionally, unilateral lesions are rare in fibromuscular dysplasia but common with carotid webs. Atherosclerosis 
Wojcik, $K$

A

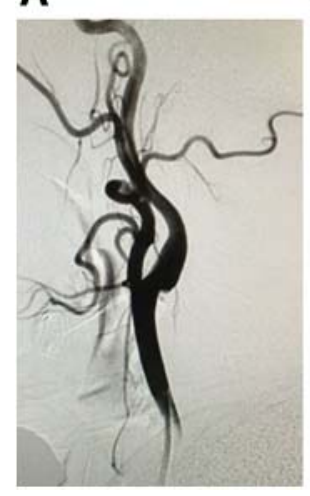

B

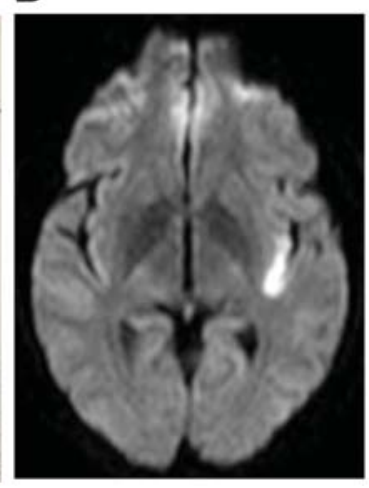

C

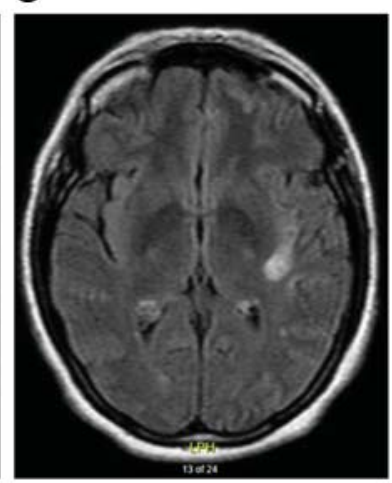

Figure 6. Case 5, a 51-year-old female with acute middle cerebral artery stroke. (A) Digital subtraction angiogram shows a shelf-like filling defect at the level of the carotid bulb. (B) Diffusion-weighted imaging shows restricted diffusion within the posterior left insular cortex with corresponding (C) T2/FLAIR (fluid attenuation inversion recovery) hyperintensity compatible with acute infarct.

typically appears on axial CT images as concentric calcification or hypoattenuation, with or without associated luminal narrowing. Heavy, circumferential calcification can cause beam-hardening artifacts that can result in overestimation of stenosis. Notably, atherosclerotic narrowing can cause elevated velocities on Doppler ultrasound, but this finding is typically absent in the setting of nonstenotic carotid webs. Additionally, a potential pitfall that could obscure imaging of carotid webs involves potential dephasing artifacts on MRA.

\section{CAROTID WEBS AS A NIDUS FOR RECURRENT ISCHEMIC STROKES}

Carotid webs have been gaining consideration as a source for stroke in young patients who otherwise have few or no risk factors for stroke. In our case series, the patient who had suffered multiple strokes (Case 1) had reoccurring embolic events in the same vascular distribution (Figure 2). The recurring location and ipsilateral nature of the carotid web suggest the plausibility that carotid webs may be a nidus for these recurrent ischemic strokes. Sajedi et al reported similar findings; nearly all patients with stroke who had carotid webs had them ipsilateral to the stroke (with 3 having bilateral webs). ${ }^{3}$ The authors proposed that the presence of a carotid web produces stasis of blood flow distal to the web, resulting in thrombus formation. ${ }^{3}$ The thrombus may then dislodge and embolize intracranially, ${ }^{1}$ thereby explaining the connection between the carotid webs identified in these patients and the occurrence of strokes despite their extraordinarily low risk profiles and further supporting the hypothesis that carotid webs are likely contributory to, if not causative of, cryptogenic strokes.

\section{DEGREE OF STENOSIS NOT SIGNIFICANT MEASURE OF STROKE RISK}

It is well established that the risk of ischemic stroke ipsilateral to atherosclerotic carotid stenosis increases as the degree of atherosclerotic stenosis increases, as outlined by the North American Symptomatic Carotid Endarterectomy Trial (NASCET) criteria. $^{11-13}$ However, these established guidelines are not likely applicable to carotid webs as the pathogenesis differs. Therefore, carotid web models for management and intervention will likely be distinct from atherosclerosis models. Albeit with small sample sizes, Dhruv et al showed that stroke patients who were found to have carotid webs displayed a nearly equal distribution of degree of stenosis. ${ }^{14}$ This finding further suggests that the degree of stenosis in carotid webs is not a significant measure of stroke risk.

\section{CURRENT AND FUTURE MANAGEMENT}

No current guidelines exist for carotid webs, and despite reports of different management strategies, ${ }^{15}$ including stent placement, ${ }^{16}$ the optimal management strategy is unknown. Carotid webs can be categorized as symptomatic (producing strokes and/or TIAs) or as asymptomatic that are identified incidentally. Management for these two categories is debated. When considering the thrombogenic theory, medical management in the form of antiplatelet and/or anticoagulation therapy represents an antithrombogenic management plan similar to that for atrial fibrillation. Given the grave prognosis and potential for recurring embolic events from symptomatic webs, a more invasive management approach involving either stenting or carotid endarterectomy may be preferred. The follow-up management of patients receiving these interventions is also debatable. Dual antiplatelet therapy vs anticoagulation vs both and length of time for medical management postintervention are only a few issues that have not yet been studied.

\section{CONCLUSION}

Carotid webs represent an underrecognized etiology for stroke, particularly cryptogenic strokes in younger patients who often do not have typical stroke risk factors. In recent years, increased awareness and improved vascular imaging techniques have greatly contributed to the increase in diagnosis of carotid webs. However, no management guidelines currently exist, and thus optimal management remains debated. 


\section{ACKNOWLEDGMENTS}

The authors have no financial or proprietary interest in the subject matter of this article.

\section{REFERENCES}

1. Choi PM, Singh D, Trivedi A, et al. Carotid webs and recurrent ischemic strokes in the era of CT angiography. AJNR Am J Neuroradiol. 2015 Nov;36(11):2134-2139. doi: 10.3174/ajnr. A4431.

2. Momose KJ, New PF. Non-atheromatous stenosis and occlusion of the internal carotid artery and its main branches. Am J Roentgenol Radium Ther Nucl Med. 1973 Jul;118(3):550-566.

3. Sajedi PI, Gonzalez JN, Cronin CA, et al. Carotid bulb webs as a cause of "cryptogenic" ischemic stroke. AJNR Am J Neuroradiol. 2017 Jul;38(7):1399-1404. doi: 10.3174/ajnr.A5208.

4. Kliewer MA, Carroll BA. Ultrasound case of the day. Internal carotid artery web (atypical fibromuscular dysplasia). Radiographics. 1991 May;11(3):504-505.

5. Karlson KJ, Wolf B, Neptune WB. Symptomatic carotid stenosis secondary to an intraluminal web-a case report. Vasc Endovasc Surg. 1987 Nov;21(6):422-426. doi: 10.1177/ 153857448702100609.

6. McNamara MF. The carotid web: a developmental anomaly of the brachiocephalic system. Ann Vasc Surg. 1987 Dec;1(5):595597. doi: 10.1016/S0890-5096(06)61448-9.

7. Haussen DC, Grossberg JA, Bouslama M, et al. Carotid web (intimal fibromuscular dysplasia) has high stroke recurrence risk and is amenable to stenting. Stroke. 2017 Nov;48(11):31343137. doi: 10.1161/STROKEAHA.117.019020.

8. Finsterer J. Management of cryptogenic stroke. Acta Neurol Belg. 2010 Jun;110(2):135-147.
9. Plouin PF, Perdu J, La Batide-Alanore A, Boutouyrie P, GimenezRoqueplo AP, Jeunemaitre X. Fibromuscular dysplasia. Orphanet J Rare Dis. 2007 Jun 7;2:28. doi: 10.1186/1750-1172-228.

10. Slovut DP, Olin JW. Fibromuscular dysplasia. N Engl J Med. 2004 Apr 29;350(18):1862-1871. doi: 10.1056/NEJMra032393.

11. den Hartog AG, Achterberg S, Moll FL, et al; SMART Study Group. Asymptomatic carotid artery stenosis and the risk of ischemic stroke according to subtype in patients with clinical manifest arterial disease. Stroke. 2013 Apr;44(4):1002-1007. doi: 10.1161/STROKEAHA.111.669267.

12. Ballotta E, Da Giau G, Meneghetti G, Barbon B, Militello C, Baracchini C. Progression of atherosclerosis in asymptomatic carotid arteries after contralateral endarterectomy: a 10-year prospective study. J Vasc Surg. 2007 Mar;45(3):516-522. doi: 10.1016/j.jvs.2006.11.011.

13. Rockman CB, Riles TS, Lamparello PJ, et al. Natural history and management of the asymptomatic, moderately stenotic internal carotid artery. J Vasc Surg. 1997 Mar;25(3):423-431.

14. Dhruv P, Kim J, Koffel J, Anderson D, Streib C. Abstract 207: A systematic literature review of patients with carotid web and acute ischemic stroke. Stroke. 2017;48(Suppl 1):A207. http:/ /stroke.ahajournals.org/content/48/Suppl_1/A207.short.

15. Elmokadem AH, Ansari SA, Sangha R, Prabhakaran S, Shaibani A, Hurley MC. Neurointerventional management of carotid webs associated with recurrent and acute cerebral ischemic syndromes. Interv Neuroradiol. 2016 Aug;22(4):432-437. doi: $10.1177 / 1591019916633245$.

16. Martinez-Perez R, Lownie SP, Pandey SK, Boulton MR. Stent placement for carotid web. World Neurosurg. 2017 Feb;98:879. e9-879.e11. doi: 10.1016/j.wneu.2016.11.050. 